\title{
A Robust Image Processing Algorithm for Optical-Based Stress-Strain Curve Corrections after Necking
}

\author{
Feng Lu, Tomáš Mánik, Ida Lægreid Andersen, and Bjørn Holmedal
}

\author{
Submitted: 5 January 2021 / Revised: 24 March 2021 / Accepted: 27 March 2021 / Published online: 24 May 2021
}

\begin{abstract}
To determine the stress-strain curve of a ductile material up to the fracture from a tensile test, the necking contour is measured by an optical measurement technique. The radius of the minimal cross-sectional area and the radius of curvature are used as input for analytical necking corrections of the stress-strain curve, as well as for finite element inverse simulations. Due to the increasing surface roughness that develops during testing, a precise determination of the specimen contour is very challenging. This is crucial, since the second derivative is required for estimating the radius of curvature. A dedicated contour-tracking algorithm was developed to deal with the surface roughness and a specimen painted white with black background was found to provide enough contrast. The new algorithm was implemented in a software, which is made available as open source. Tests were made for an isotropic, commercially pure aluminum alloy and for an axisymmetric, peak aged AA6082 alloy, based on image recording by a digital camera and synchronized force measurements. Modeling by finite element simulations was performed to assess the accuracy of analytical corrections of the stress-strain curves by inverse modeling and for designing a robust contourtracking algorithm.
\end{abstract}

Keywords axisymmetric tensile test, digital image analyses, mechanical testing, mechanical system, modeling and simulation, necking correction, stress-strain relation

\section{Introduction}

The precise determination of the stress-strain relationship up to large strains is one of the challenges for accurate modeling of several forming operations involving large strains, as well as for the precise characterization of ductility and plasticity of nanostructured metals (Ref 1,2$)$. The equivalent stress-strain curve can easily be derived for the uniform tensile deformation up to the onset of diffuse necking in a tensile test. After this point, there will be a triaxial stress mode with nonuniform pressure distribution across the necking region. The measurement of the stress-strain curve after the onset of necking is far more complex, as the geometry of the neck must be accounted for ( $\operatorname{Ref} 3)$.

Commonly, modern tensile machines are equipped with cameras. These are used for digital image correlations to estimate strains during testing. By recording the necking, they can also be used for the purpose of making necking corrections and thereby extend the strain range obtained by the tensile test. A robust algorithm is then required to extract the information about the necking contours from the images.

Inverse modeling analysis using finite element (FE) simulations is the general method for making stress corrections due

Feng Lu, Tomáš Mánik, Ida Lægreid Andersen, and Bjørn Holmedal, Department of Materials Science and Engineering, Norwegian University of Science and Technology, NO-7491 Trondheim, Norway. Contact e-mail: bjorn.holmedal@ntnu.no. to necking, see, e.g., for a specimen with round cross section (Ref 4, 5), for a rectangular cross section (Ref 6, 7), and works on notched specimens (Ref 8-11). The strength of the inverse modeling approach is that it can deal with anisotropic materials, complex cross sections and notched geometries. However, with increased complexity and accuracy, a complex calibration of an anisotropic yield surface is required for precise FE simulations, and the mesh must be sufficiently fine. Furthermore, the inverse modeling needs well-defined targets based on measured quantities that are to be matched by the simulation. Matching the measured change of the force and the minimum crosssectional area as functions of the post-necking elongation do not always correspond to only one possible stress-strain curve. Ideally, a more precise measured neck geometry should also be a target, and the weighting of the different targets must be carefully chosen. Such an inverse model requires many simulations to be run in an iterative manner to determine the stress-strain curve of the model, requiring a considerable computing time.

Alternatively, simplified analytical solutions can be applied for the necking correction. Such solutions exist for an isotropic material and an axisymmetric specimen geometry. The classic analytically derived correction factor by Bridgman (Ref 12), for estimating the stress-strain curve after onset of necking, is based on a simplified solution. Similar analytical solutions have been proposed (Ref 13-15) applying slightly different mathematical simplifications to derive first-order analytical approximate solutions. Gromada et al. (Ref 16) recently proposed a necking correction based on a higher order, more complex, analytical solution. In common for all these analytical solutions is that their corresponding correction factors require measured radius of the minimal cross-sectional area $a$, and radius of curvature of the necking contour $R$, as input, i.e., these two must be measured during the test.

As an alternative to measuring $R$, Bridgman (Ref 17) suggested to measure only $a$ and use an experimental observed approximate relation between $a / R$ and the strain. Based on 
tensile tests of steels, bronze and brass, Bridgman tabulated and plotted such a relation for tensile tests of axisymmetric bars up to $400 \%$ strain, suppressing fracture by a high hydrostatic pressure. It is noted from his results that for $\varepsilon \leq 1$, his curve is almost linear. He also reported more detailed results for necking of thin-walled tubes, for which he reported a linear relationship $a / R=0.83\left(\varepsilon-\varepsilon_{u}\right)$. Later, Le Roy et al. (Ref 18$)$ reported a similar approximately linear relationship $a / R=1.1\left(\varepsilon-\varepsilon_{u}\right)$ for the case of solid samples made of carbon steels. This relation can be applied, e.g., in combination with the Bridgman correction (Ref 19, 20). The measurement of a may be performed during a tensile test, e.g., by a sweeping laser-based extensometer (Ref 21-23) or by image-based measurements (Ref 24-26). Tu et al. (Ref 27) measured the necking contours during tensile testing in two orthogonal plane mirrors, using one camera. Their contour tracking algorithm was not sufficiently precise to estimate the curvature radius of the contour $R$, instead estimated they used the simplified Le Roy approximation.

Vilamosa et al. applied a high-speed camera for recording Split-Hopkinson tension tests at elevated temperatures (Ref 28). The images were analyzed with an edge detection algorithm by Hopperstad et al. (Ref 24). La Rosa et al. (Ref 29) evaluated the influence of the approximations in the Bridgman necking correction method by comparing FE modeling and experimental results from camera-based measurements of the necking profiles. A. Sancho et al. (Ref 30) applied a similar setup with a camera recording images of a specimen being painted white with a black background. Here, Bridgman's method was used for necking corrections, where the radius of curvature of the contour was estimated from the pictures by fitting osculating circles to the contours. A complex approach by Siegmann et al. (Ref 31 ) estimated both $a$ and $R$ with an advanced setup, applying a high-resolution camera, utilizing fringe projection as well as digital image correlation, acquiring three-dimensional geometry information of the necking region. When needing to make necking corrections to obtain precise stress-strain curves, the present authors found that the quality of the setup and the algorithms were not sufficiently well documented in the works so far, which motivates this work, where also a corresponding open source software with a user friendly graphical user interface is made available for this purpose, see (Ref 32).

External cameras or built-in cameras in the tensile test machine have high resolution and enough image quality and are no longer the bottle neck of image-based measurements of necking during tensile testing. However, even with sharp images at perfect light condition, the surface of the specimen will be roughened during testing, mainly due to the deformation, making the contour tracking increasingly challenging as the strain increases. The main challenge is to estimate $R$, which requires a smooth estimate of the second derivative of the contour in the neck. The main goal of this work is to design a robust and precise image processing algorithm that can be applied to extract contours from each necking image, recorded by one or more cameras during testing. It aims at measuring not only $a$, but also $R$ with enough accuracy for the purpose of necking corrections. Hence, the stress-strain curve can be estimated up to fracture.

The paper is organized as follows: Section 2 provides a description of the experimental setup. In section 3, the variations of $a$ and $R$ are estimated by a finite element model of the tensile test. Simulation results are used for aiding the design of the algorithms for precise extraction of contour information from the images. The FE model is also applied for inverse simulations. Section 4 explains the detailed procedures of extracting the specimen contours from the images in order to estimate $a$ and $R$, from which the analytical necking corrections can be performed. In section 5, the methodology is applied to tensile tests for a soft, ductile commercially pure aluminum alloy and for a strong, peak hardened AA6082 aluminum alloy with earlier onset of necking. An assessment of the classical method by Bridgman (Ref 12,17) and the recent method by Gromada (Ref 16) are made, where the accuracy of neckingcorrected results is discussed in terms of the FE simulations and in terms of the experimental error sources. Finally, conclusions are made in section 6 .

\section{Experimental Procedures}

\subsection{The Tensile Test Specimen}

A commercially pure aluminum alloy (wt.\% $=99.7 \%$ ) received from Hydro Aluminum as a DC-cast round extrusion ingot with $228 \mathrm{~mm}$ diameter, and an extruded AA6082 round bar with $30 \mathrm{~mm}$ diameter, received from Neuman Aluminum Raufoss, were the materials used in this investigation. Tensile test specimens were machined from the center region of both the round ingot and the extruded bar, with tensile axis in the axial direction. Figure 1 illustrates the cylindrical tensile test specimen, where the parallel part has a cross-sectional diameter of $6 \mathrm{~mm}$ and a length of $18 \mathrm{~mm}$. Note that this length is shorter than according to the standard (Ref 33). The reason for this was to obtain an optimal image resolution. An alternative approach when measuring longer specimens, would be to machine a tapered specimen, i.e., impose a slight thickness variation to trigger the necking at a given position.

The commercially pure aluminum specimens were heated at a rate of $100^{\circ} \mathrm{C} / \mathrm{h}$, homogenized at $550^{\circ} \mathrm{C}$ for 6 hours and air cooled afterward. This did not affect the random as-cast texture. The extruded AA6082 specimens were solution heat treated in a salt bath at $540^{\circ} \mathrm{C}$ for 12 minutes, then water-quenched to room temperature. After 10 minutes storage at room temperature, the samples were artificially aged in an oil bath for 3 hours (peak aged) at $180^{\circ} \mathrm{C}$, and subsequently water cooled. Due to the manganese-based dispersoids, the extruded AA6082 alloy did not recrystallize during the heat treatments, and still had the axisymmetric fiber texture from the extrusion, illustrated by the inverse pole figure in Fig. 2, measured using an XRD goniometer in a Siemens D5000 XRD machine and calculated using the Bruker Texeval 2.5 software and the MATLAB toolbox MTEX. For more details, see, e.g., the book by Randle and Engler (Ref 34 ).

\subsection{Experimental Setup and Data Acquisition}

Figure 3 illustrates the experimental setup for image and force-data acquisition during the tensile testing. A Walter + Bai multipurpose Servo hydraulic universal testing machine was used. A National Instrument multifunctional I/O device was used for converting the analogue force signal into a digital signal and transferring it to a computer. A high-resolution $5120 \times 3840$ monochrome CMOSIS CMV20000 mono 5K industrial camera was employed for image acquisition. The side of each pixel measured about $5 \mu \mathrm{m}$. The camera was equipped with a Canon EF $100 \mathrm{~mm}$ f/2.8L Macro IS USM lens. A large- 
bandwidth fiber cable connected the camera to the computer for transferring raw image data.

Good light conditions are important to ensure a high image quality with evenly distributed light on the specimen to ensure clear contrast when tracking the specimen contours. The light conditions and the quality of the image need to remain stable during the test. A black PVC photographic background sheet was used as a backdrop in the testing machine, as illustrated in Figure 3. For enhancing the contrast between the specimen and the background, the tensile specimen was painted using "Hard Hat Topcoat Matt White 2190" spray from Rustoleum. The painting had a good ductility and deformed along with the specimen without cracking and without losing adhesion when the surface roughness increased during deformation. The camera was mounted on a tripod in front of the specimen, and the focus was optimized for the distance to the two edges of the specimen. Two DC-powered white LED panels equipped with white light diffusers, were applied as light sources, being symmetrically mounted, providing equal light on both sides of the specimen. The specimen was vertically aligned using a laser leveler. The specific position where the necking occurs is not known in advance. Hence, the entire parallel length was included in the picture, taking into account some increased length for the elongation of the specimen during the test (for details, see section 4.2).

Both black and white painting of the specimen were tested, with white and black background, respectively, see Fig. 4. Due to the reflections of the light by the specimen, the round specimen may have a bright zone close to its contours, as seen in Fig. 4(b). Since this makes the border bright, a dark background is beneficial for identifying the contour. A combination of black background and white specimen provided optimal contrast.

The test was started by that a control signal was sent from the computer, both to the camera and to the force acquisition port. The image data and the digital force signal were acquired simultaneously at the same frequency of $1 \mathrm{~Hz}$. The maximum image acquisition frequency of the system was $120 \mathrm{~Hz}$. Tensile tests were conducted at room temperature with a crosshead speed of $0.54 \mathrm{~mm} / \mathrm{min}$, corresponding to a nominal strain rate of $5 \cdot 10^{-4} \mathrm{~s}^{-1}$, which is typical for tensile testing, and which provides time for capturing images during the test. Approximately 900 images were captured for the AA6082 alloy, and 2500 images for the commercial pure aluminum during each test. There is one image for each point on the stress-strain

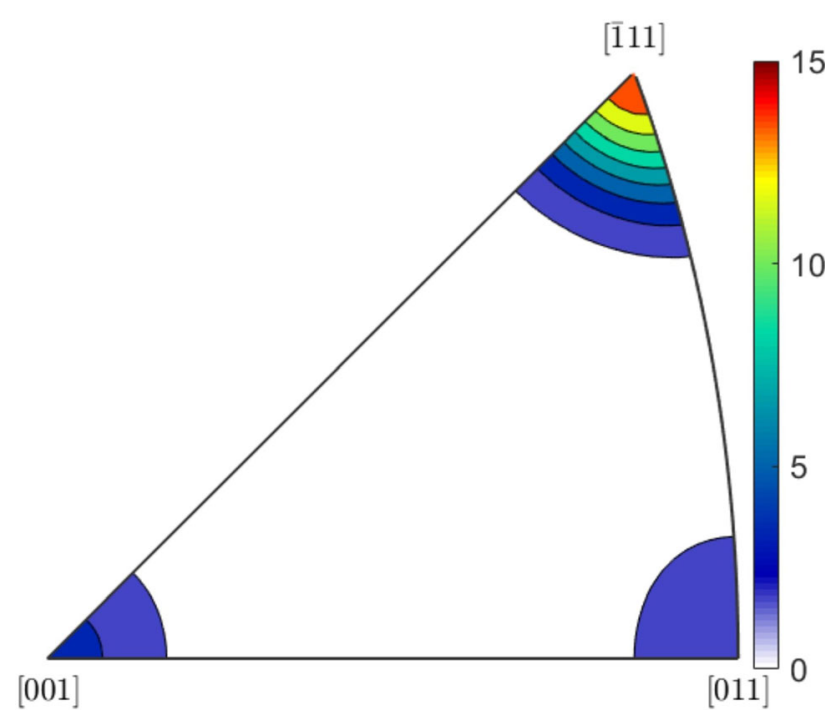

Fig. 2 Inverse pole figure of the extruded and peak aged AA6082 alloy. The color map shows the extrusion axis direction, where the value indicates multiples of random distribution

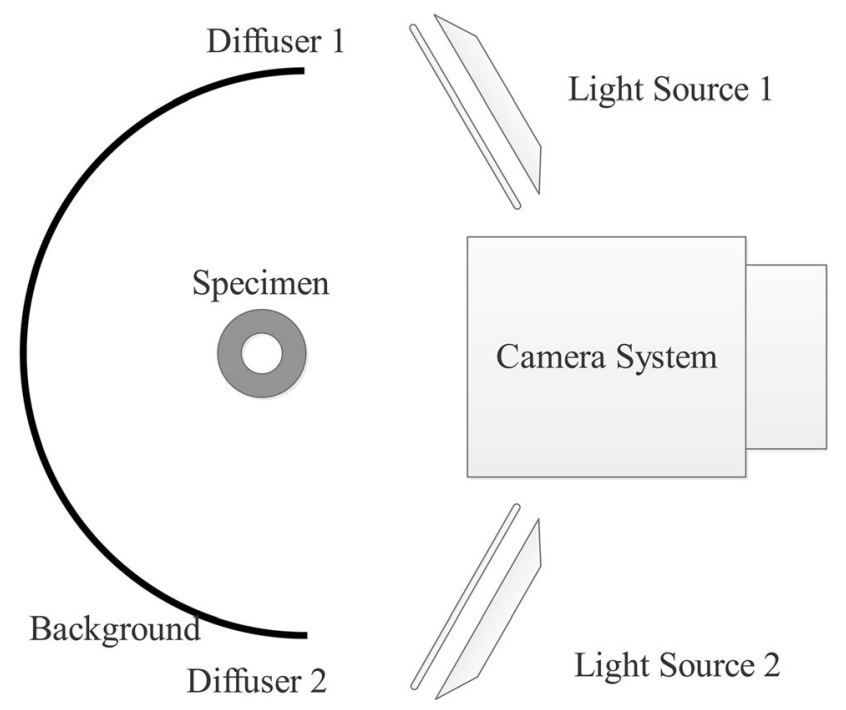

Fig. 3 Schematic diagram of the uniaxial tensile test setup, seen from above

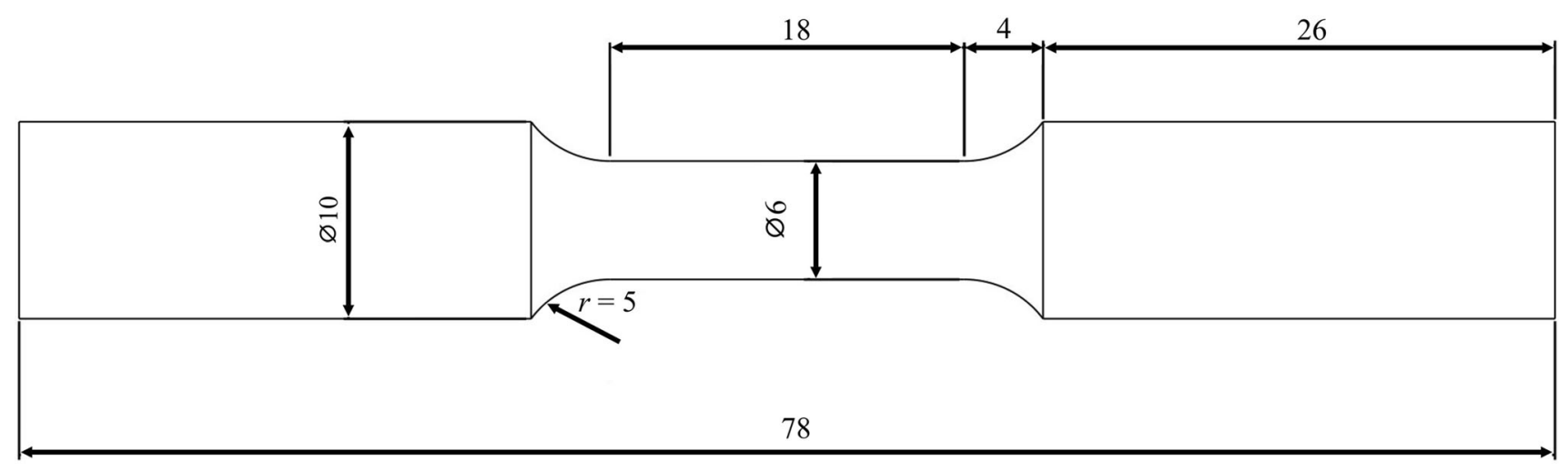

Fig. 1 Geometry of the specimens for uniaxial tensile tests (measured in $\mathrm{mm}$ ) 
curve. In most cases, fewer points than this will be sufficient for a good resolution of the stress-strain curve.

\section{FE Simulations}

A uniaxial tension test of a cylindrical specimen was simulated using the finite element software Abaqus Standard 2017. Figure 5(a) shows the mesh for the specimen in an axisymmetric model with a stress-free outer surface. Nodes at one end were fixed, while the nodes on the opposite end had a prescribed velocity along the tensile axis but were free to move radially. The dimensions of the FE model are given in Fig. 1. The gauge region was meshed with linear four-node axisymmetric elements, CAX4, applying full integration. Elements of type CAX3 were used for the rest of the tensile specimen. The element size was $5 \times 150 \mu \mathrm{m}^{2}$, resulting in 3600 elements along the tensile direction and 20 elements in radial direction. The element length in the tensile direction corresponds to the typical pixel size in the pictures taken by the camera. For the inverse modeling (section 3.2), the element size was increased to $50 \times$ $150 \mu^{2}$ in order to increase the computational efficiency, while not compromising the accuracy. Isotropic elastoplastic material behavior was modeled, using Young modulus $E=70 \mathrm{GPa}$ and Poisson's ratio $v=0.3$ for the elastic part. The rate insensitive von Mises model, with or without isotropic hardening, was applied for the plastic part. See section 3.2 on more details on the work hardening law used and the way inverse modeling was performed.

Figure 5(c-d) shows a simulated contour of a well-developed neck in a simulation of pure aluminum, with the corresponding distribution of the plastic von Mises strain shown in Fig. 5(b). In principle, for a given contour, the minimum specimen radius, $a$, is found as the minimum distance between the contour and the center axis. The radius of curvature of the contour can be calculated by

$R=\frac{\left(1+y^{\prime 2}\right)^{\frac{3}{2}}}{\left|y^{\prime \prime}\right|}$

Here, $y^{\prime}(x)$ is the first derivative and $y^{\prime \prime}(x)$ is the second derivative of the contour line. As seen in Fig. 5(e), the radius of curvature $R$ is smallest at the center of the neck and increases rapidly with increasing distance from this point. In the necking correction procedures, it is this minimum $R$ that is of interest; hence, it is very important to have a precise description of the specimen contour line.

The use of the FE model in this work is twofold. Firstly, it is used to improve the algorithm for calculating the radius $R$ by

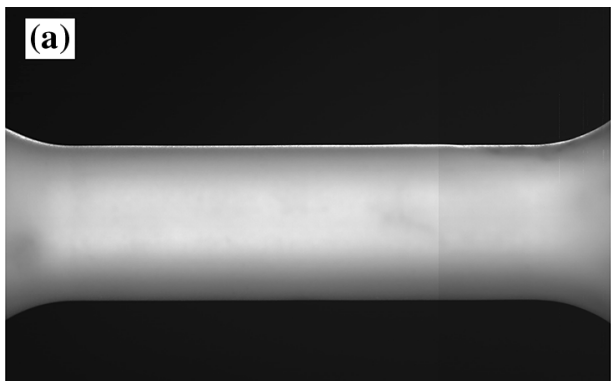

analyzing the error made by the contour fitting algorithm. Secondly, inverse modeling of the material tensile curve is performed based on the least-square fit of the measured stress, $F / \pi a^{2}$

\subsection{The Fitting Length $L$ Required to Estimate $R$}

In order to calculate the radius $R$ in the thinnest part of the neck, a second-order polynomial was fitted for each measured strain, in the least-square sense, to a contour interval of a certain axial length $L$, containing the minimum thickness of the neck. The estimated radius $R$ is sensitive to the fitted length $L$. For the ideal, smooth and noise-free contour from an FE simulation, a very short $L$ can be applied, providing a precise estimate of $R$.

Figure 6 shows how $R$, estimated from the simulated contour, increases with increasing $L$. For this calculation, the model was applied without work hardening, which gives fastest development of the neck. For real contours containing noise and other structural irregularities, a too short fitting interval $L$ will lead to poor determination of $R$. This can clearly be seen from the red curve in Fig. 6, where additive white Gaussian noise with a standard deviation of $5 \mu \mathrm{m}$, similar to the measured scattering, was superposed to the smooth simulated contour. The same polynomial fit was made as for the smooth contour.

With increasing $L$, on the other hand, $R$ is increasingly overestimated, as seen from Fig. 6. The FE model was used to assess the increase of $R$ with increased fitted region . Choosing very low $L$ would lead to a result strongly affected by the noise of the contour. Choosing a larger $L$ will make the fit less sensitive to the noise at the expense of an overestimated $R$. The maximum length, $L_{10 \%}$ for which the overestimation of $R$ was less than $10 \%$, was calculated as a function of the strain. Curves for $L_{10 \%}$ and $L_{3 \%}$ and are compared in Fig. 7. It is clear from Fig. 6 that $L_{10 \%}$ is a suitable choice when the noise is controlled by the pixel size, as in this case. A higher image resolution may allow a stricter curve, e.g., $L_{3 \%}$, while a larger may be required when the surface is rougher.

\subsection{Inverse Simulation of the Uniaxial Tensile Test}

In order to assess the performance of the proposed experimental technique, inverse modeling of the material work-hardening model was carried out using the FE model. The target to be matched by the simulations, in a least-square sense, was the measured force divided by the minimum crosssectional area as a function of the logarithmic strain. The strain was based on the minimum cross-sectional area of the neck and was measured for strains up to fracture. Von Mises plasticity was applied, and an extended Voce hardening law, given in Eq. 2, was fitted to represent the work hardening of the

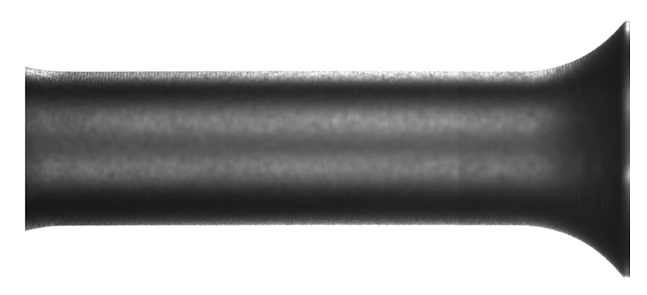

Fig. 4 Light conditions and reflections with (a) dark background and white specimen and (b) white background and black specimen 

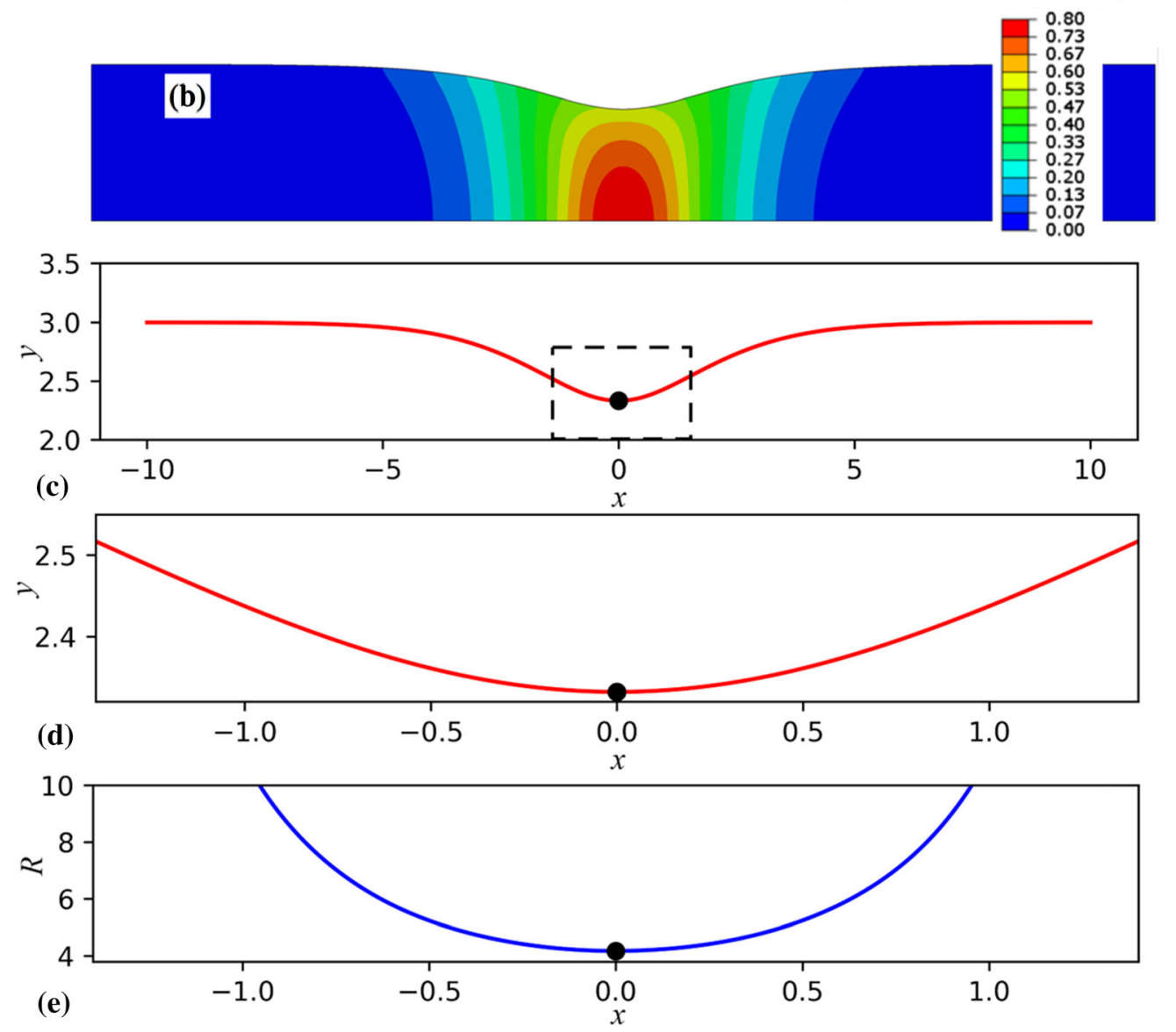

Fig. 5 (a) Finite element mesh of the gauge area, upper symmetric part; FE simulation at a tensile strain of 0.5. (b) von Mises plastic equivalent strain. (c) The surface contour of the specimen in (b). (d) A close up of the selected contour from (c). (e) The radius of curvature $R$ as a function of $x$ for the contour in (d)

material. However, the work hardening in Abaqus is represented by a piece-wise linear stress-strain curve through points specified in a table. Hence, instead of using the Abaqus optimization software, the Powell's hybrid optimization algorithm was run in a python script, from which tabulated stressstrain curves were fitted to the Voce curves as input to Abaqus simulations, which were run iteratively by the script. The force and the minimum cross-sectional area of the neck were returned by Abaqus, and the script modified the stress-strain curve to optimize the fit of the measured, uncorrected stress as a function of the logarithmic strain, as shown in Fig. 8. The fitted parameters of the extended Voce hardening law are given in Table 1.

$$
\begin{aligned}
R(\varepsilon)= & R_{0}+R_{1}^{s}\left(1-\exp \left(-\frac{\varepsilon}{\varepsilon_{1}^{s}}\right)\right)+R_{2}^{s}\left(1-\exp \left(-\frac{\varepsilon}{\varepsilon_{2}^{s}}\right)\right) \\
& +\theta \varepsilon
\end{aligned}
$$

\section{Processing the Images}

A python code was programmed to process the images and the corresponding force data. The program runs an algorithm to extract the contours from the bitmap images. In the region close to the minimum specimen thickness in the neck, a polynomial is fitted to the contour, from which the radius of the minimal cross-sectional area, $a$, and the radius of curvature, $R$, of the necking-contour are estimated from each image. The detailed algorithms are described below.

\subsection{Images Pre-Processing}

The first image after loading and the latest image before fracture were first cropped manually, in order to remove the specimen heads from the images, see Fig. 9(a) and (d). One of the ends of the specimen is moving during elongation; hence, the neck is moving relative to the image area. The position of the thinnest part of the neck is identified at the last image before fracture; then, its position is traced back to the onset of necking. 


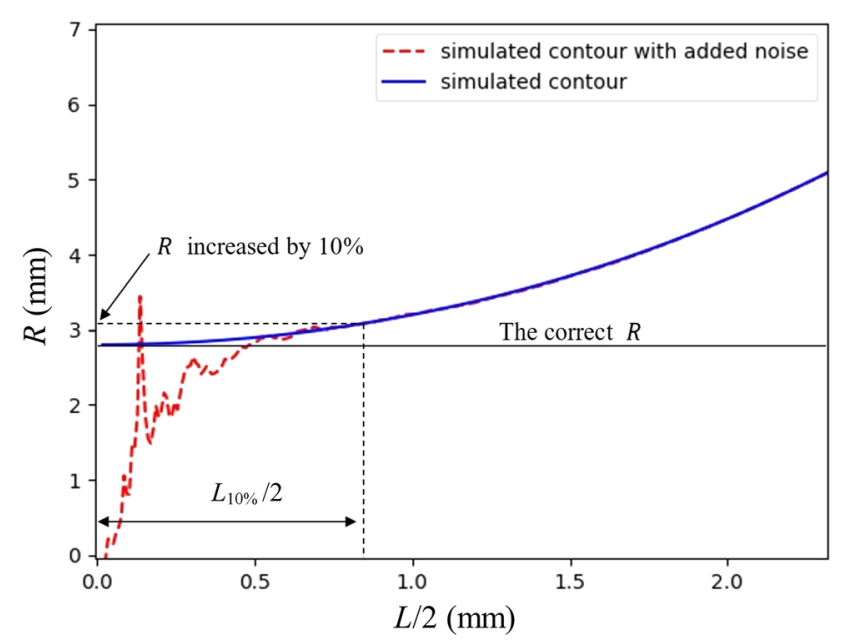

Fig. 6 Radius of curvature $R$ as a function of the fitting interval length $L$ at a strain of 0.67 . The red curve is calculated from the smooth simulated contour with additive white Gaussian noise with standard deviation of $5 \mu \mathrm{m}$

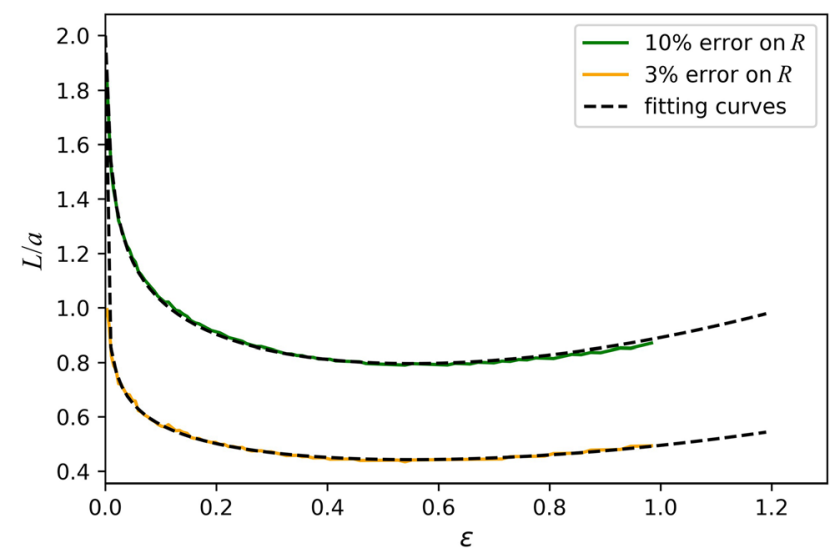

Fig. 7 Fitting region $L$, with $10 \%$ and $3 \%$ error on the radius $R$, determined from the simulation of the rigid plastic case. The curves fitted by Eq. (3) are dashed

The images are cropped to an identical image size, so that the whole neck is always within the images, as illustrated in Fig. 9.

\subsection{Contour Tracking Algorithm}

The black background is utilized to determine the specimens' outer contour. To avoid single white spots on the black background, a median filter with a $5 \times 5$ pixels window was firstly applied to the images. The distortion of the specimen edge by this median filter is small as compared to the surface roughness and does very little affect the further identification of the edge. All lines, of one-pixel width, in the radial direction across the specimen, are considered. An example is illustrated by the blue line in Fig. 10(a). The gray-scale intensity in the picture is in the range from zero (black) to 256 (white). In Fig. 10(b) the intensity distribution along the blue line in Fig. 10(a) is shown. For each such line the intersections with the two contours of the specimen must be identified, in the transition between the black low-intensity background and the brighter specimen. In the following, an algorithm is suggested to identify the pixel position of the two borders of the specimen

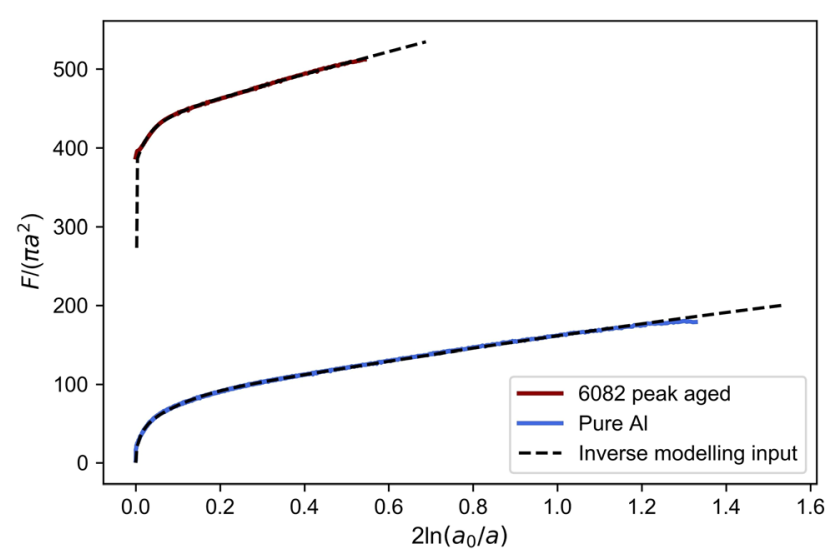

Fig. 8 Measured force as function of the logarithmic strain based on the measured minimum cross-sectional radius $a$, for the pure aluminum and for the AA6082. The dashed curves show the calibrated FE model calculations

Table 1 Fitted parameters of the extended Voce hardening law for both pure aluminum and AA6082

\begin{tabular}{lll}
\hline Voce parameters & Pure aluminum & AA6082 \\
$R_{0},(\mathrm{MPa})$ & 379.7 & 16.5 \\
$R_{1}^{s},(\mathrm{MPa})$ & 68.3 & 57.1 \\
$R_{2}^{s},(\mathrm{MPa})$ & -27.9 & 28.8 \\
$\varepsilon_{1}^{s}$ & 0.037 & 0.177 \\
$\varepsilon_{2}^{s}$ & 0.411 & 0.018 \\
$\theta,(\mathrm{MPa})$ & 6.9 & 164.9 \\
\hline
\end{tabular}

for each line across the specimen for a given image. The basic idea is to estimate the background intensity level of the black region outside the specimen and perform a line search inward to identify the first pixel that has a gray-scale intensity that significantly deviates from the background level outside.

Firstly, a rough estimate is made for the radial position corresponding to the transition between the black background and the white specimen. For this purpose, the maximum intensity along each transverse pixel line is found by a scan through all its pixels, i.e., along the $y$-direction in Fig. 10(a) (the blue line). Next, two line searches are performed, starting from each their outer end of the transverse line and moving pixel by pixel inward toward the tensile specimen. The accumulated average intensity of all pixels scanned so far on the way inward toward the specimen is used as the estimate of the background intensity. Once the next pixel probed exceeds this level by more than a factor $f_{1}$ times the difference between the maximum intensity level and the background intensity level, this pixel provides the first estimate of the contours' $y$ location, and the scan is terminated. Since the transition between the black background and the white specimen is quite sharp, the range $0.1<f_{1}<0.7$ will provide sufficiently good first estimates. In this work $f_{1}=0.2$ is applied. The two resulting points on each side of the specimen are shown as green points on the scanned line in Fig. 10(b). The locations of these two points along the $\mathrm{y}$-axis are denoted $y_{1}^{(1)}$ and $y_{1}^{(2)}$. The first estimate of the diameter of the specimen along the considered line is $d_{1}=\left|y_{1}^{(2)}-y_{1}^{(1)}\right|$.

Next, a more refined estimate of the background intensities at the two sides is made. The first estimate of the contour 

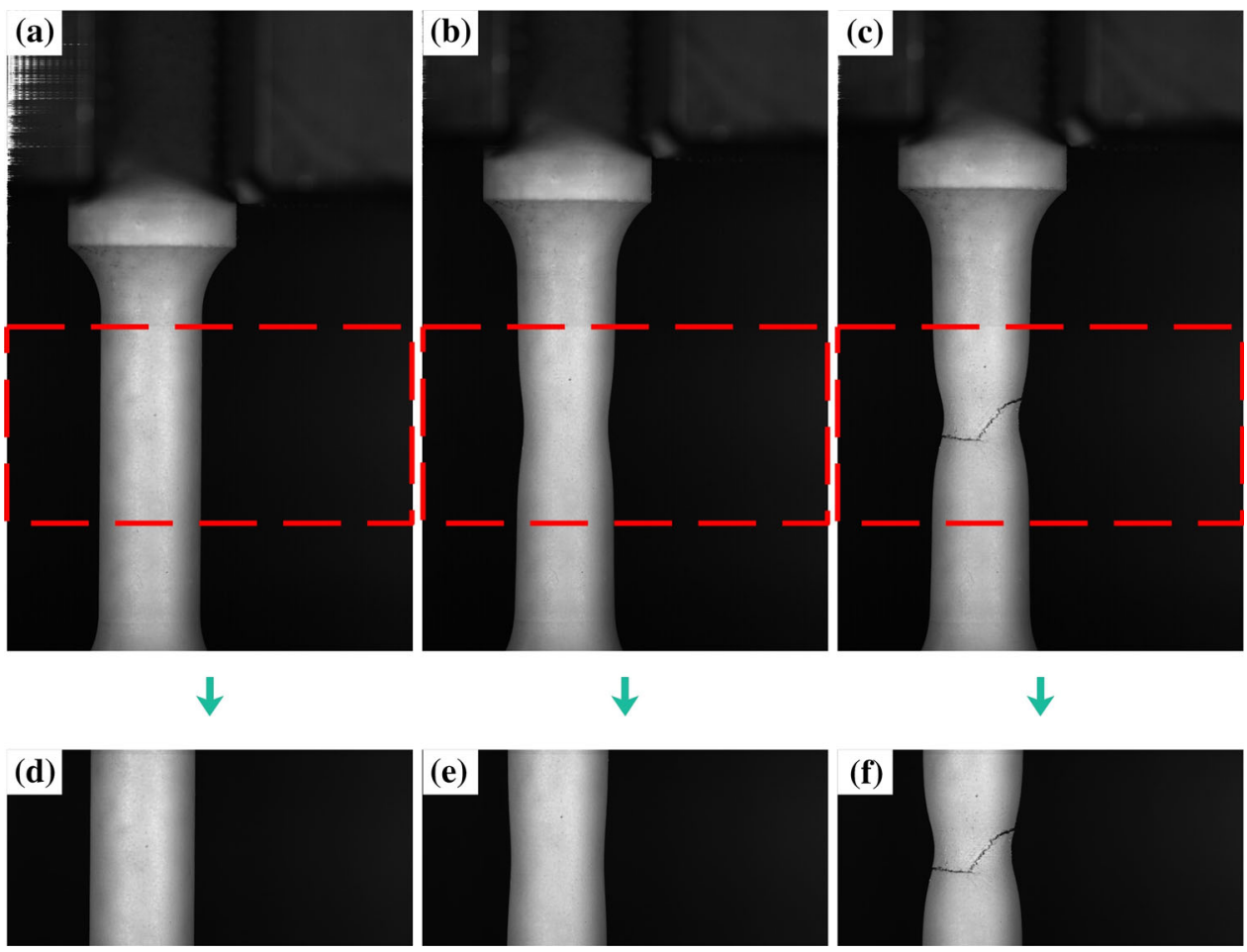

Fig. 9 Images from the AA6082 test, (a) and (d) show uniform deformation before necking (b) and (e) show a pronounced neck and (c) and (f) show the specimen right after fracture. (d-f) show the cropped images used to extract the contours

location is used to narrow the region required for estimating the background level intensity. The nearest regions outside the first estimated contour lines are excluded by disregarding a small length, $f_{2} d_{1}$ outside each side of the firstly estimated contour locations. The factor $f_{2}$ must be sufficiently large to capture the background level, $0.05<f_{2}<1$ will give about similar results. In this work $f_{2}=0.2$ is applied. A length equal to the first estimate of the specimen diameter $d_{1}$ is now considered for estimating the background intensity on each side of the specimen. A least-square fit of a linear trend line for the intensity as a function of $y$ is made in this interval. The standard deviations of the background intensities in these intervals are calculated. To ensure a level significantly above the background intensity, the trend lines are shifted upward by a factor $f_{3}$ times the difference between the maximum intensity and the average background intensity of the trend line within the considered interval. Ideally, $f_{3}$ should be as small as possible but sufficiently large to exceed the noise level. In this work $f_{3}=$ 0.08 was applied. A larger value might be required in cases where the noise of the background level is stronger. The trend lines and the shifted ones are shown as green lines in Fig. 10(c). To avoid detecting pixels caused by noise in the background region, it is ensured that the lines always are shifted at least five times their standard deviations.

New refined estimates of the contour borders are obtained similar as for the first estimates, by searching inward along the transverse line. However, the search can now start quite close to the specimen, from the innermost position in the region where the background level was fitted, i.e., a distance $f_{2} d_{1}$ outside each side of the firstly estimated contours. Once the intensities of the next two pixels both exceed the level given by the shifted trend line, an interpolation between the next pixels point and the considered one is made, and its crossing point with the shifted trend line is determined. These locations, i.e., the two red points in the close up in Fig. 10(c), are taken as the improved final estimates of the contour locations $y^{(1)}$ and $y^{(2)}$ for the considered blue line at axial position $x_{i}$ in Fig. 10(a), where $i$ is the pixel number in the axial direction in the image.

Now, for a given image, i.e., a given tensile strain, the two contour lines along the tensile axis, i.e., the $x$-direction, can be found by the collection of all contour points from all transverse radial lines. An example of such contour lines is plotted in Fig. 11.

\subsection{Specimen Radius and Radius of Curvature of the Contour}

For strains up to necking, the parallel region of the tensile specimen remains uniform. At the uniform strain, before necking has occurred, a small rotation of the reference coordinate system is made if required, ensuring that the average direction of the two straight contour lines is parallel with the $x$-axis. All contour lines are then estimated in this coordinate system.

The contour lines extracted from the images, consist of a collection of points and are not completely smooth. Smoothed first and second derivatives are required for calculating $a$ and Rmust be estimated numerically. This can be achieved by an interpolating polynomial curve that is fitted by the least square method. A high-order polynomial will give high accuracy for a smooth curve, but it is sensitive to oscillations due to influence of the noise. A second order polynomial has a constant second derivative, but the fitted interval of length $L$ must be small in order to obtain the desired accuracy. The latter approach is more robust and was chosen. The normalized length $L / a$ required for a satisfactory accuracy of $R$, varies with increasing 

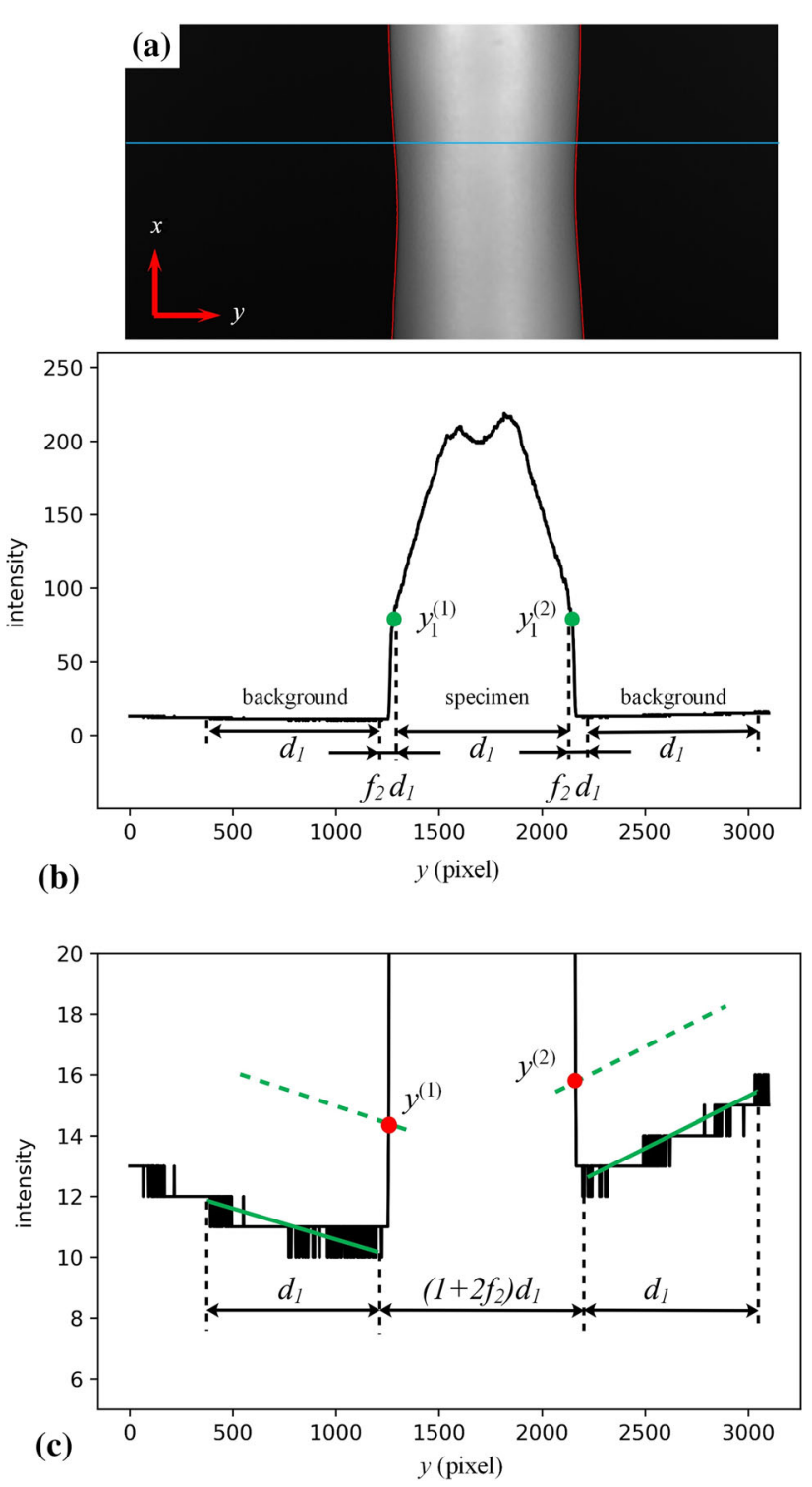

Fig. 10 (a) Example of estimated contours. (b) The intensity along the blue transverse line in (a) with the first estimate of the contours by the proposed algorithm. (c) A close up of (b), showing the final determination of the contour points as the interceptions with the shifted, dashed line of the background noise

post-uniform strain, $\varepsilon-\varepsilon_{u}$ as the neck becomes more pronounced with increasing strain, where $\varepsilon$ is the equivalent strain, and $\varepsilon_{u}$ is the strain at onset of necking, corresponding to the maximum load. This was investigated by finite element simulations of a tensile test without work hardening, The following expression, with $f_{4}=1$, fits well the curve for the $10 \%$ error as shown in Fig. 7 in section 3.1.

$\frac{L}{a}=\min \left(2, f_{4}\left(1.579\left(\frac{1}{\varepsilon-\varepsilon_{u}}\right)^{\frac{1}{10}}+0.2754\left(\varepsilon-\varepsilon_{u}\right)^{2}-0.963\right)\right), \varepsilon>\varepsilon_{u}$

Curves with different errors of $R$ scales with this relation, e.g., the curve with $3 \%$ error of $R$ is very close with a factor of $f_{4}=0.56$. Both curves are shown in Fig. 7. In this work, $f_{4}=1$ was applied for peak aged AA6082, while $f_{4}=1.5$ was applied for the pure aluminum, due to the higher ductility with more noise from the deformed surface in the neck at the largest strains.

Knowing the fitting length from Eq. (3), the estimates for $a$ and $R$ were conducted for each image. Each sample has one contour on each side, which are treated as two contours with the centerline in common. The contours were extracted from bitmap-formatted images with the number of image pixels as unit. The following procedure was used to estimate $a$ and $R$ at the neck for one contour:

1. A first search provides an estimate of the minimum diameter by identifying the pixel line $x_{i}$ which corresponds to the smallest distance $\min \left(\left|y^{(1)}-y^{(2)}\right|\right)$ between its two contours. The corresponding axial position is $x_{\min }$. However, right after necking the position of the neck is very difficult to determine. In such cases, when $\varepsilon-\varepsilon_{u}<0.05$, the first guess for the axial position, $x_{\min }$, is back extrapolated from the images at slightly higher strains.

2. Based on the estimated necking position, $x_{\min }$, a second order polynomial is fitted to each of the contour line point representations $y^{(1)}$ and $y^{(2)}$ in the interval $x_{\min } \pm L / 2$. For each of the two contours, an improved estimate $y_{\min }^{(1,2)}$ for and the corresponding position $x_{\min }$ are made by the minimum points of the two fitted polynomials. If the new minimum is at one of the ends of the fitted polynomial, step 2 is repeated based on this position as an updated estimate for. However, one iteration is in most cases enough. The radius is in common for the two contours and is estimated as $a_{L}=\left|y_{\min }^{(2)}-y_{\min }^{(1)}\right| / 2$. The radius of curvature $R_{L}$ is calculated individually for each contour from its second order polynomial, at each their minimum point.

3. Based on the estimate of the necking position from step 2 , a new second order polynomial is fitted to each contour line in a twice as large interval $x_{\min } \pm L$. Corresponding estimates of the radius and the radius of curvature $R_{2 L}$ for each contour follow from these second order polynomials.

4. The estimate for $a / R$ converges quadratically with decreasing $L / a$. Hence, if this relation is smooth, an improved estimate can be obtained by extrapolation to $\mathrm{L}=$ 0 .

$\frac{a}{R}=\frac{a_{L}}{R_{L}}+\frac{1}{3}\left(\frac{a_{L}}{R_{L}}-\frac{a_{2 L}}{R_{2 L}}\right)$

5. It is expected that $a_{2 L} / R_{2 L}<a_{L} / R_{L}$. However, in cases where the noise is influencing the estimate, sometimes this does not hold. In such cases, the estimate based on the smallest range is influenced by the noise and must be rejected and $a / R=a_{2 L} / R_{2 L}$ is used instead.

The change of units from number of pixels to physical dimensions requires that the pixel size is precisely determined. This is done by that the specimen average diameter is measured before the tensile test, and the corresponding average number of pixels between the two specimen contour lines is found from the first captured image. The estimated $a$ and $R$ from the images were converted from pixels to millimeters, allowing the stress to be calculated. 

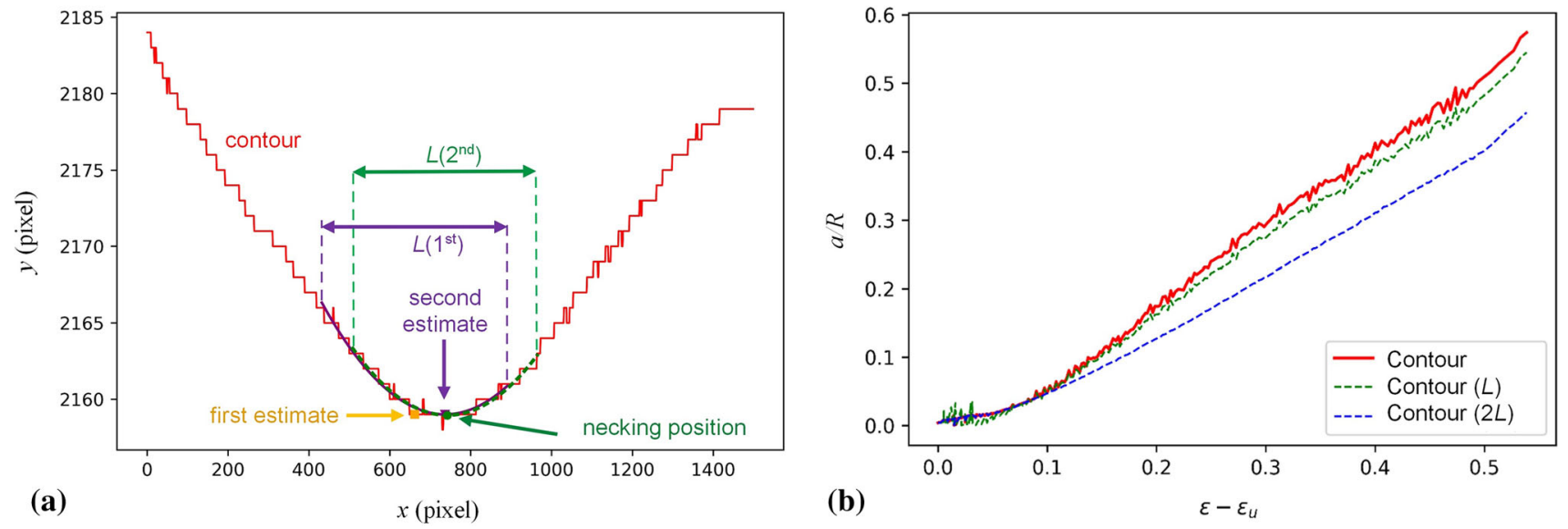

Fig. 11 (a) Necking position obtained by a polynomial of order 2 fitted on a contour interval of length $L$. (b) $a / R$ as function of $\varepsilon-\varepsilon_{u}$. In both (a) and (b) the green dashed curve is obtained with an interval $L$, the blue dashed curve with interval $2 L$, and the red curve by extrapolation toward zero $L$ (Color figure online)

\subsection{The Strain}

The transverse strain component in the neck is calculated from the radius $a$ as $\varepsilon_{r r}=\ln \left(a_{0} / a\right)$, where $a_{0}$ is the initial radius of the specimen However, the strain of interest is the equivalent strain, i.e., $\varepsilon=\varepsilon_{z z}=\ln \left(l / l_{0}\right)$, which is the strain that are commonly measured in uniaxial tensile tests (without necking), wherein $l$ is the elongated length of an extensometer of initial length $l_{0}$ This strain $\varepsilon_{z z}$, can be decomposed into an elastic part $\varepsilon_{z z}^{\mathrm{el}}$ and a plastic part $\varepsilon_{z z}^{\mathrm{pl}}$.

When the strain is small, the contribution from the elastic part of the strain is significant. Hooks law of elasticity is assumed

$\varepsilon_{z z}=\varepsilon_{z z}^{p l}+\varepsilon_{z z}^{e l}=\varepsilon_{z z}^{p l}+\frac{\sigma_{z z}}{E}$

$\varepsilon_{r r}=\varepsilon_{r r 1}^{p l}+\varepsilon_{r r 2}^{e l}=\varepsilon_{r r}^{p l}-v \frac{\sigma_{z z}}{E}$

Here, $E$ is Young's modulus and $v$ is Poisson's ratio. For axisymmetric specimens, the radial plastic strain $\varepsilon_{r r}^{\mathrm{pl}}$ will be equal in all transverse directions. The trace of the plastic strain tensor vanishes due to volume conservation.

$\varepsilon_{z z}^{p l}+2 \varepsilon_{r r}^{p l}=0$

It follows from Eq. (5), (6) and (7) that

$\varepsilon_{z z}=(1-2 v) \frac{\sigma_{z z}}{E}-2 \varepsilon_{r r}$

The elastic strain component should be included at small strains close to the elastic transition

$\varepsilon_{z z}=(1-2 v) \frac{F}{E \pi a^{2}}-2 \ln \left(\frac{a}{a_{0}}\right)$

At larger strains, the elastic strain component is neglectable and $\varepsilon=\varepsilon_{z z} \approx 2 \ln \left(a_{0} / a\right)$.

\subsection{Stress Corrections}

From the onset of necking the stress triaxiality changes in the necking region, and the changed distribution of the hydrostatic pressure must be accounted for. Based on a simplified analytical solution, Bridgman (Ref 12) suggested the following stress correction.

$\sigma=\frac{F}{\pi a^{2}\left(1+\frac{2 R}{a}\right) \ln \left(1+\frac{a}{2 R}\right)}$

Recently, a more complex simplified solution was proposed by (Ref 16), being based on a higher-order series expansion of the displacement field in the neck.

$$
\begin{aligned}
\sigma= & \frac{F}{\pi a^{2} \xi} \\
\xi= & \frac{9}{7}-\frac{5 \Lambda}{7(1+5 \Lambda)}-\frac{2(1-6 \Lambda)}{7(1+5 \Lambda)}+\frac{30 \Lambda(8 \Lambda-\delta-5 \delta \Lambda)}{49 \delta(1+5 \Lambda)^{2}}+\frac{3(8 \Lambda-\delta-5 \delta \Lambda)}{7 \delta(1+5 \Lambda)} \\
& \left(\frac{2(1-6 \Lambda)}{7(1+5 \Lambda)}-\frac{2}{7}-\frac{30 \Lambda(8 \Lambda-\delta-5 \delta \Lambda)}{49 \delta(1+5 \Lambda)^{2}}\right) \ln \left|1+\frac{7(1+5 \Lambda)}{3(8 \Lambda-\delta-5 \delta \Lambda)}\right|
\end{aligned}
$$

$\delta=\frac{a}{R}, \Lambda=1-\frac{a_{0}}{a}$

\section{Results and Discussion}

The necking-correction methodology was implemented into an open source software (Ref 32) and tested for two alloys. The DC-cast and homogenized commercially pure aluminum alloy had random grain orientations, which was confirmed by x-ray diffraction, and the stress is therefore isotropic. This alloy was expected to have considerable work hardening and a high local fracture strain in the neck. The extruded AA6082 had the fiber texture shown in Fig. 2, hence the stress is axisymmetric. In the peak hardened condition, it is expected to have limited work hardening and to fracture at a lower local strain in the neck.

The measurement of the elastic part of the stress-strain curve of the peak aged AA6082 alloy is not a target by this technique. Small elastic strains can precisely and more easily be measured by an extensometer. However, it is calculated and shown in Fig. 12 to show the accuracy of the strain measurements by this image-based technique. Since the 
elasticity of aluminum alloys is nearly isotropic, Young's modulus was not iteratively determined from the measured stress-strain curve, but rather $E=70 \mathrm{GPa}$ was applied in the strain calculation by Eq. (9). As shown in Fig. 12, the measured curve fits reasonably well the measured elastic part of the curve, confirming the validity of the guessed Young's modulus. The image resolution allows the radius to be resolved by about 500 pixels; hence, the error seen in Fig. 12 corresponds to measuring the radius within one-pixel size precision, i.e., approximately $5 \mu \mathrm{m}$. Compared to the initial radius, this corresponds to a strain of about $10^{-3}$. Compared to the large strains beyond necking, for which this technique is developed, this is a very small error.

The estimation of the radius of curvature $R$, from the image data, requires the second derivative of the contour line, which is sensitive to noise. The radius of curvature was estimated based on the images, both for the left and the right contour of the same specimen. For one selected test of each alloy, the resulting two $a / R$ curves for each side of the specimen, are shown in Fig. 13(a) and (c), respectively.

In addition, inverse FE modeling results are included in the plots in Fig. 13. Note, that the stress-strain curves were calibrated by the inverse modeling to obtain the measured force-elongation curve, while neither the radii nor the detailed specimen contour were calibrated. The resulting calibrated model parameters are listed in Table 1. For the AA6082 alloy, necking occurred at $\varepsilon_{u}=0.06$. For this case, the inverse modeling follows the measured $a / R$ to a strain $\varepsilon-\varepsilon_{u} \approx 0.35$. At larger strains than this, the measured $a / R$ points are slightly lower than from the simulation. The two curves from each side of the specimen are very similar, indicating that the noise due to surface roughness is not significantly influencing the result in this case.

The difference between equivalent and uncorrected stressstrain curves is strikingly for the AA6082 in Fig. 13(b), for which the FE simulation and the equivalent curve with Gromada's formula show softening, while the uncorrected curve shows significant work hardening. It is assumed that the FE simulation here provides the best estimate. An approximately flat stress-strain curve beyond the uniform strain for AA6082 in the peak aged condition was recently reported also by (Ref 35), performing well-lubricated compression tests. Note that the Bridgman correction is not sufficient, showing a weak work hardening in this case. In Fig. 13(b), the Bridgman correction gives a difference of approximately $7 \%$ as compared

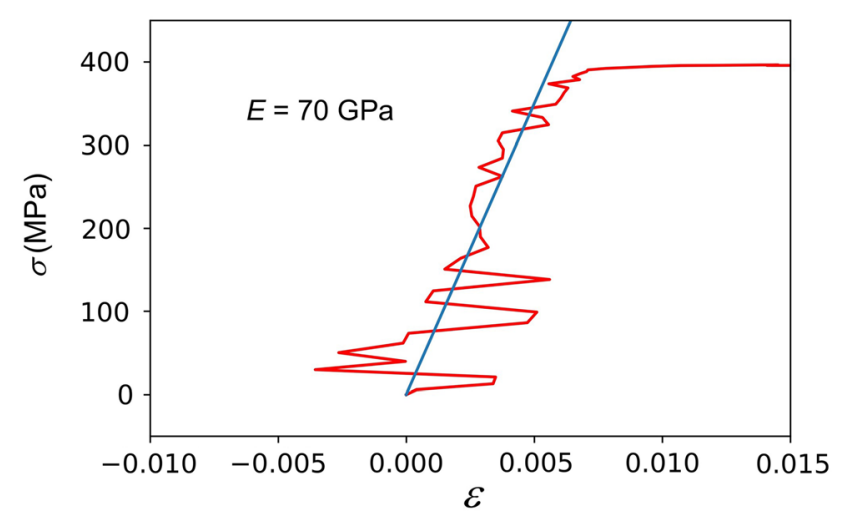

Fig. 12 Experimentally obtained elastic part of the stress-strain curve of the AA6082 alloy to the inverse modeling at a strain $\varepsilon \approx 0.5$, while Gromada's correction gives less than $3 \%$ difference. Furthermore, the stress-strain curve in Fig. 13(b) has the same shape as from FE simulation with Gromada's correction. This correction is close to the inverse simulation result and therefore significantly better than the Bridgman correction.

For the pure aluminum specimen, necking occurred at a larger strain, $\varepsilon_{u}=0.29$, and the measured $a / R$ curves from the two contours of the same specimen in Fig. 13(c) do not overlap precisely. Still, they follow the inverse modeling curve reasonably well, within the experimental spread, up to a strain of $\varepsilon \approx 1$. At larger strains, the inverse modeling estimates a slightly smaller slope of the $a / R$ curve than can be seen for the one estimated from the images. Since the specimen is axisymmetric, the spread between the $a / R$ curves for the two contours of the same specimen is mainly caused by the surface roughness.

It is interesting to note the almost linear asymptotic behavior of $a / R$ as function of strain in Fig. 13, for both cases, similar as reported by Bridgman at even larger strains, and with a slope close to 1.1, as proposed by Le Roy et al. However, in both cases, a transition in behavior occurs between the onset of necking at the uniform strain and the asymptotic linear relationship at larger strains. Note that this transition and the linear Le Roy asymptotic behavior of the $a / R$ curves can also be seen for other metals in the works by La Rosa et al. (Ref 29) and by Siegmann et al. (Ref 31). For the AA6082 alloy, this transition occurs after the onset of necking during a strain interval up to $\varepsilon-\varepsilon_{u} \approx 0.1$, while for the pure aluminum it lasts until $\varepsilon-\varepsilon_{u} \approx 0.7$. Only after this nonlinear transition, $a / R$ follows the linear asymptotic relation, until fracture occurs. The difference between the two alloys in the shape of the $a / R$ curves is related to the difference in the post-necking work hardening and is verified by the inverse simulations, also included in Fig. 13.

Note the difference in ductility. The peak aged AA6082 fractures $\varepsilon_{f} \approx 0.55$ at while the commercially pure aluminum fractures at $\varepsilon_{f} \approx 1.3$. At this strain, damage evolution, e.g., voids, is expected to contribute to lowering the stress. The specimen surface roughness increases with increasing strain. Due to the large uniform and fracture strains of the commercially pure alloy, its contours suffer from a significant amount of noise. Close to fracture, the necking contour is strongly influenced by the surface distortions. This is illustrated in Fig. 14(b), showing an image of the neck at a strain of $\varepsilon \approx 1.1$, taken for the one among the parallel tests with the largest difference $a / R$ of between its two contours, as seen in Fig. 14(a). In general, the larger strain for this alloy results in a significantly larger experimental spread of the $a / R$ curves than for the peak aged AA6082, as seen in Fig. 15, where all measured curves are included. At the largest strains, as in Fig. 15(b), the required fitting length $L$ becomes similar to the typical wavelength of the surface noise, which lowers the precision. As seen from the influence of the simulated noise in Fig. 6, the radius can randomly be over- or underestimated with a too small value of $L$.

Similar as for the analytical corrections, an isotropic material is assumed in the FE modeling. This is strictly valid only for the commercially pure aluminum. The analytical solutions, on which Bridgman's and Gromada's corrections are based, only consider the stress mode and the hydrostatic pressure distribution in the smallest cross section of the neck. 

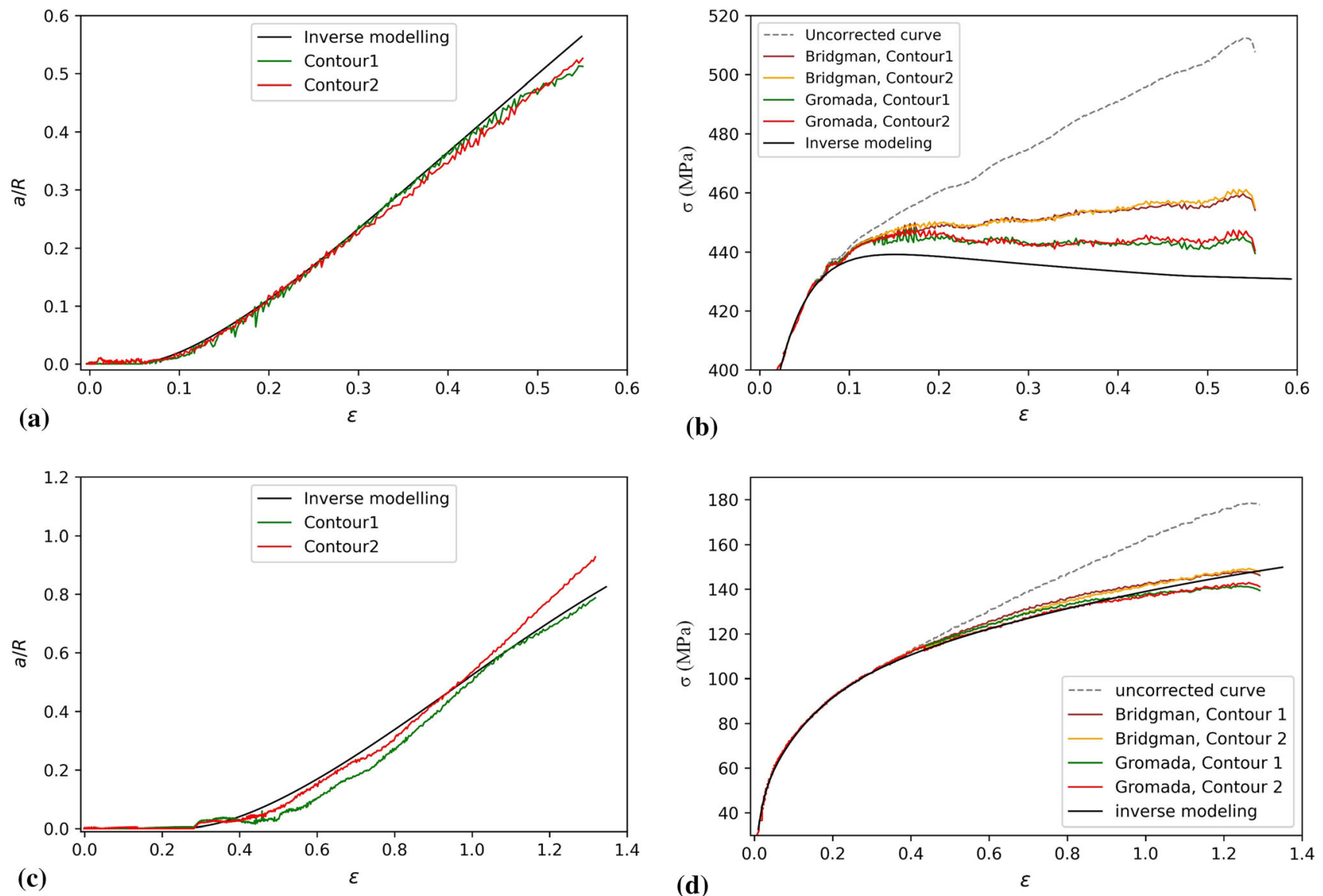

Fig. 13 Comparison of experimental and inverse modeling ratio $a / R$ versus equivalent strain of (a) peak aged AA6082 alloy and (c) pure aluminum. Comparison on Bridgman and Gromada corrected equivalent stress-strain curves and inverse modeling result of (b) peak aged AA6082 alloy, and (d) pure aluminum

Here the loading mode remains uniaxial tension, also during necking. Hence, the same loading mode is the case in these regions both before and after necking, i.e., the solution remains mainly on the same point on the yield surface. However, in the regions away from the minimum cross section of the neck, shear strains are invoked. In this region, an axisymmetric material, or an isotropic material with a higher yield surface exponent than the quadratic von Mises criterion, might respond differently than the isotropic von Mises model used in the FE simulation, influencing the shape of the neck. This is a higher order effect that is not included in the FE simulations of the AA6082. However, the close match of $a / R$ to the experiments in Fig. 15(a) indicates that the radius is predicted well with the isotropic assumption, also for the axisymmetric tests.

While the analytical methods are restricted to materials without sensitivity to strain rate and temperature, such dependencies can be included in an FE simulation. A proper calibration would then require additional material tests. The simulations of the pure aluminum show that the strain rate in the neck became about ten times higher in the neck at the largest strains obtained in the test. Since the strain rate sensitivity for aluminum is low at room temperature, corrections were not made. In a well conducting material as aluminum, there will be time for the heat diffusion away from the neck and the temperature will not rise much during testing at low strain rates, while in high-speed tests deformation heating might soften the neck. Precautions should be made in such cases, which were not covered by this work.

Stress-strain curves for all parallel tests performed are shown in Fig. 15(c). The experimental spread is reasonably low, within a few percent for both alloys. It can be seen from Fig. 13(d) and Fig. 15(c), that for the curves of pure aluminum, Gromada's correction provides stress-strain curves following the inverse simulation up to a strain of about unity. From Fig. 13(d), it is realized that the Bridgman correction is too small, hence the stress is overestimated by $5-10 \%$. At larger strains, there is a significant spread of the $a / R$ curves, as can be seen from Fig. 15(b). However, for the AA6082 case, the surface quality remains much better due to the smaller strain, hence, the $a / R$ curves in Fig. 15(a) show a small spread and match the FE curve well.

In Fig. 8, the inverse modeling approach was able to match the measured $F / \pi a^{2}$ curve very well. It also managed to capture the experimental $a / R$ behavior for both alloys in Fig. 15(a) and (b). Note that the quality of the inverse modeling relies on two important aspects. Firstly, high computational cost is necessary for the inverse model to converge, making the computation last several tens of hours (for the cases tested). Secondly, a rather advanced scripting is required to orchestrate the whole iteration process, including FE model updating, post processing and making a new guess. Figure 15 proves that the experimental method proposed here, using a camera to record 


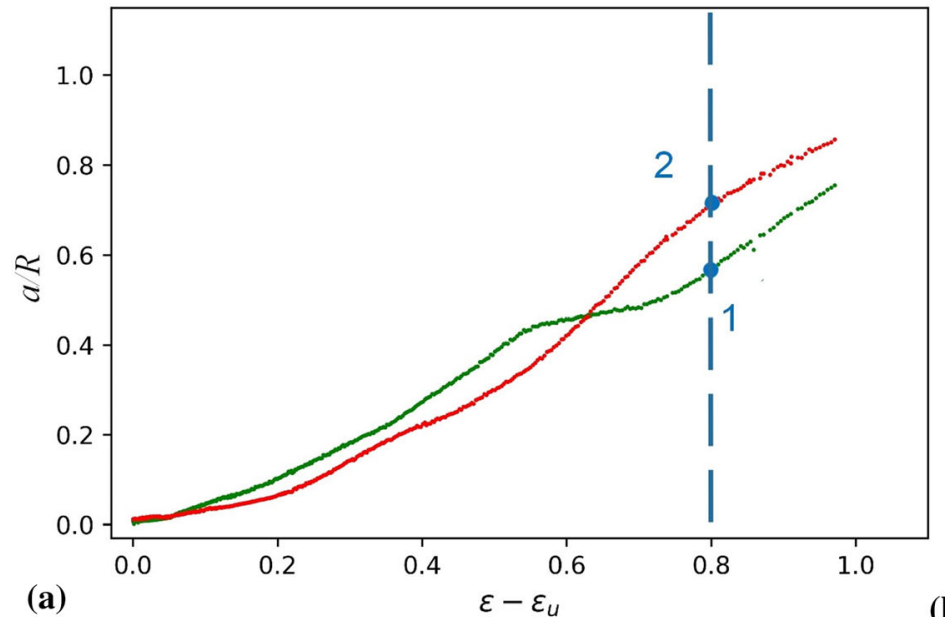

(a)

Fig. 14 (a) Ratio $a / R$ versus $\varepsilon-\varepsilon_{u}$ obtained from contour 1 and 2 of the pure aluminum specimen. (b) The necking region of the pure aluminum specimen at strain $\varepsilon-\varepsilon_{u} \approx 0.8$
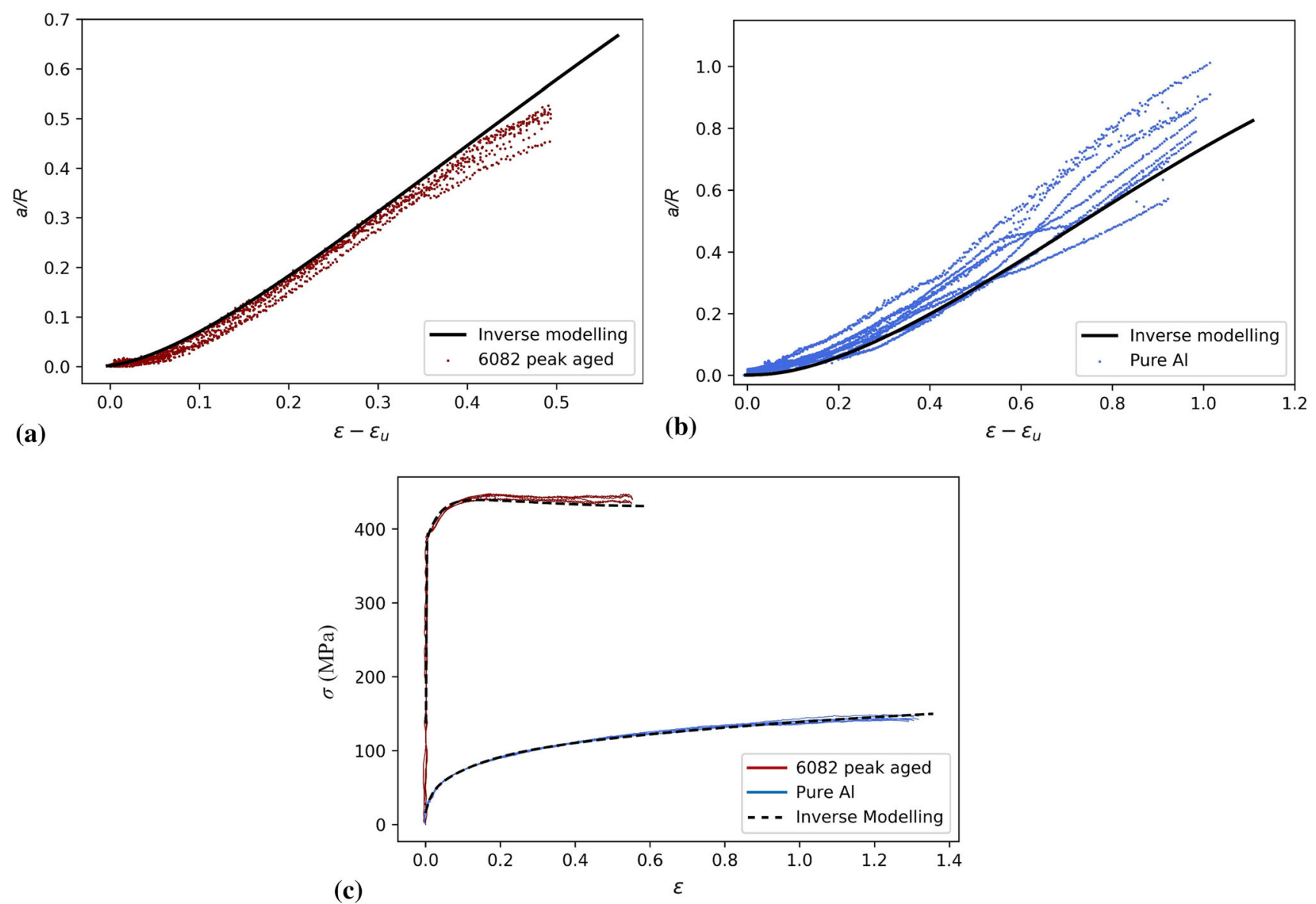

Fig. 15 Measured $a / R$ obtained from all parallel specimens of (a) the peak aged AA6082 alloy and (b) the pure aluminum. (c) Comparison of inverse modeling results and corresponding equivalent stress-strain curves by Gromada's correction for the peak aged AA6082 alloy and the pure aluminum

the tensile test and Gromada's analytical formula for the necking correction, provides a solution which is very close to the one obtained by inverse modeling. The stress-strain curve up to large strains can thus be very accurately calculated by Gromada's analytical formula by a negligible computational cost as compared to the inverse FE modeling.

\section{Conclusion}

In the present work, algorithms were developed for making precise necking corrections of the stress, based on images recorded during tensile testing of axisymmetric specimens. A 
white specimen with a black background was found to provide an optimal contrast. An algorithm is formulated for extracting the two contours of the specimen from the series of bitmap images. The contours are further processed by another algorithm, developed for estimating the minimum cross-sectional radius of the specimen and the radius of curvature of the necking contour. This algorithm was designed, based on FE simulations, to handle the noise that occurred due to limited image resolution and increasing surface roughness during the test. Inverse FE modeling was conducted to verify the accuracy and robustness of the technique and to compare and assess Bridgman's and Gromada's stress corrections during necking. The algorithms were tested for two different alloys. The commercially pure aluminum was soft, ductile, with considerable work hardening to large strains. The peak hardened AA6082 was strong, less ductile, and showed weak work softening at large strains. In both cases, Gromada's stress correction was found to be significantly closer to the $\mathrm{FE}$ simulations than Bridgman's correction.

\section{Acknowledgments}

The authors acknowledge support from the AMPERE project [NFR 247783], a knowledge-building project for industry, cofinanced by The Research Council of Norway (NFR), and the industrial partners; Hydro, Gränges, Neuman Aluminum Raufoss (Raufoss Technology), and Nexans. The authors acknowledge Hassan Moradi Asadkandi for programming the GUI for the open source software.

\section{Funding}

Open access funding provided by NTNU Norwegian University of Science and Technology (incl St. Olavs Hospital - Trondheim University Hospital).

\section{Open Access}

This article is licensed under a Creative Commons Attribution 4.0 International License, which permits use, sharing, adaptation, distribution and reproduction in any medium or format, as long as you give appropriate credit to the original author(s) and the source, provide a link to the Creative Commons licence, and indicate if changes were made. The images or other third party material in this article are included in the article's Creative Commons licence, unless indicated otherwise in a credit line to the material. If material is not included in the article's Creative Commons licence and your intended use is not permitted by statutory regulation or exceeds the permitted use, you will need to obtain permission directly from the copyright holder. To view a copy of this licence, visit http://creativecommons.org/licenses/by/4.0/.

\section{References}

1. Y.T. Zhu and X.L. Wu, Ductility and Plasticity of Nanostructured Metals: Differences and Issues, Mater. Today Nano, 2018, 2, p 15-20

2. Y. Beygelzimer, O. Prokof'eva, R. Kulagin, V. Varyukhin and S. Synkov, Measures of Ductility for UFG Materials Obtained by SPD, Mater. Sci. Forum, 2009, 633-634, p 223-230

3. S. Tu, X. Ren, J. He and Z. Zhang, Experimental Measurement of Temperature-dependent Equivalent Stress-strain Curves of a $420 \mathrm{MPa}$ Structural Steel with Axisymmetric Notched Tensile Specimens, Eng. Fail. Anal., 2019, 100, p 312-321
4. K. Komori, Simulation of Tensile Test by Node Separation Method, $J$. Mater. Process. Technol., 2002, 125-126, p 608-612

5. A. Vaz-Romero, Y. Rotbaum, J.A. Rodríguez-Martínez and D. Rittel, Necking Evolution in Dynamically Stretched Bars: New Experimental and Computational Insights, J. Mech. Phys. Solids, 2016, 91, p 216239

6. Z.L. Zhang, M. Hauge, J. Ødegård and C. Thaulow, Determining Material True Stress-strain Curve from Tensile Specimens with Rectangular Cross-section, Int. J. Solids Struct., 1999, 36(23), p 3497-3516

7. J.M. Choung and S.R. Cho, Study on True Stress Correction From Tensile Tests, J. Mech. Sci. Technol., 2008, 22(6), p 1039-1051

8. A. Valiente, On Bridgman's Stress Solution for a Tensile Neck Applied to Axisymmetrical Blunt Notched Tension Bars, J. Appl. Mech., 2001, 68(3), p 412-419

9. M. Murata, Y. Yoshida and T. Nishiwaki, Stress Correction Method for Flow Stress Identification by Tensile Test Using Notched Round Bar, $J$. Mater. Process. Technol., 2018, 251, p 65-72

10. S. Tu, X. Ren, J. He and Z. Zhang, A Method for Determining Material's Equivalent Stress-strain Curve with any Axisymmetric Notched Tensile Specimens Without Bridgman Correction, Int. J. Mech. Sci., 2018, 135, p 656-667

11. D. Priadi, C. Magny, E. Massoni, C. Levaillant and L. Penazzi, A New Tensile Test on Notched Specimens to Assess the Forming Limit Diagram of Sheet Metals, J. Mater. Process. Tech., 1992, 32(1-2), p 279-288

12. P.W. Bridgman, The Stress Distribution at the Neck of a Tension Specimen, Trans ASM, 1944, 32, p 553-574

13. N.N. Davidenkov and N.I. Spiridonova, Mechanical Methods of Testing. Analysis of the State of Stress in the Neck of a Tension Test Specimen, Am. Soc. Test. Mater., 1947, 46, p 1147-1158

14. W. Szczepinski, On the Mechanisms of Ductile Microfracture in Metals - Experimental Modeling, Arch. Mech., 1984, 36(4), p 569-586

15. S. Schwaigere and E. Siebel, Mechanics of Tensile Test, Arch Eisenhuttenwes, 1948, 19(5), p 145-152

16. M. Gromada, G. Mishuris and A. Öchsner, Correction Formulae for the Stress Distribution in Round Tensile Specimens at Neck Presence, Springer, Berlin Heidelberg, 2011

17. P.W. Bridgman, Studies in Large Plastic Flow and Fracture with Special Emphasis on the Effects of Hydrostatic Pressure, McGraw-Hill, New York, 1952

18. G. Le Roy, J.D. Embury, G. Edwards and M.F. Ashby, A Model of Ductile Fracture Based on the Nucleation and Growth of Voids, Acta Metall., 1981, 29(8), p 1509-1522

19. I. Westermann, K.O. Pedersen, T. Børvik and O.S. Hopperstad, Workhardening and Ductility of Artificially Aged AA6060 Aluminium Alloy, Mech. Mater., 2016, 97, p 100-117

20. J. Choung, Comparative Studies of Fracture Models for Marine Structural Steels, Ocean Eng., 2009, 36(15-16), p 1164-1174

21. M. Hercher, G. Wyntjes and H. DeWeerd, Non-Contact Laser Extensometer, SPIE, Industrial Laser Interferometry, 1987, p 185-191

22. P.C. Bastias, S.M. Kulkarni, K.Y. Kim and J. Gargas, Noncontacting Strain Measurements during Tensile Tests, Exp. Mech., 1996, 36(1), p $78-83$

23. X. Nie, B. Song and C.M. Loeffler, A Novel Splitting-beam Laser Extensometer Technique for Kolsky Tension Bar Experiment, J. Dyn. Behav. Mater., 2015, 1(1), p 70-74

24. O.S. Hopperstad, T. Børvik, M. Langseth, K. Labibes and C. Albertini, On the Influence of Stress Triaxiality and Strain Rate on the Behaviour of a Structural Steel Part i Experiments, Eur. J. Mech. A/Solids, 2003, 22(1), p 1-13

25. J.H. Yu, B.A. McWilliams and R.P. Kaste, Digital Image Correlation Analysis and Numerical Simulation of Aluminum Alloys under Quasistatic Tension after Necking using the Bridgman's Correction Method, Exp. Tech., 2016, 40(5), p 1359-1367

26. C. Defaisse, M. Mazière, L. Marcin and J. Besson, Ductile Fracture of an Ultra-high Strength Steel under Low to Moderate Stress Triaxiality, Eng. Fract. Mech., 2018, 194, p 301-318

27. S. Tu, X. Ren, T.A. Kristensen, J. He and Z. Zhang, Study of Lowtemperature Effect on the Fracture Locus of a 420-MPa Structural Steel with the Edge Tracing Method, Fatigue Fract. Eng. Mater. Struct., 2018, 41(8), p 1649-1661

28. V. Vilamosa, A.H. Clausen, E. Fagerholt, O.S. Hopperstad and T. Børvik, Local Measurement of Stress-strain Behaviour of Ductile 
Materials at Elevated Temperatures in a Split-Hopkinson Tension Bar System, Strain, 2014, 50(3), p 223-235

29. G. La Rosa, A. Risitano and G. Mirone, Postnecking Elastoplastic Characterization: Degree of Approximation in the Bridgman Method and Properties of the Flow-stress/true-Stress Ratio, Metall. Mater. Trans. A, 2003, 34(3), p 615-624

30. A. Sancho, M.J. Cox, T. Cartwright, C.M. Davies, P.A. Hooper and J.P. Dear, An Experimental Methodology to Characterise Post-necking Behaviour and Quantify Ductile Damage Accumulation in Isotropic Materials, Int. J. Solids Struct., 2019, 176-177, p 191-206

31. P. Siegmann, C. Alén-Cordero and R. Sánchez-Montero, Experimental Approach for the Determination of the Bridgman's Necking Parameters, Meas. Sci. Technol., 2019, 30(11), p 114003

32. F. Lu, H.M. Asadkandi, T. Mánik, I.L. Andersen, and B. Holmedal, Necking Correction Open Source Software, https://gitlab.com/ntnu-ph ysmet/necking-correction, 2020
33. A. International, ASTM E8/E8M - 09 Standard test methods for tension testing of metallic materials, ASTM, 2011

34. V. Randle and O. Engler, Introduction to Texture Analysis : Macrotecture, Gordon \& Breach, Microtexture and Orientation Mapping, Amsterdam, 2000

35. W. Moćko, J.A. Rodríguez-Martínez, Z.L. Kowalewski and A. Rusinek, Compressive Viscoplastic Response of 6082-T6 and 7075-T6 Aluminium Alloys under Wide Range of Strain Rate at Room Temperature: Experiments and Modelling, Strain, 2012, 48(6), p 498-509

Publisher's Note Springer Nature remains neutral with regard to jurisdictional claims in published maps and institutional affiliations. 